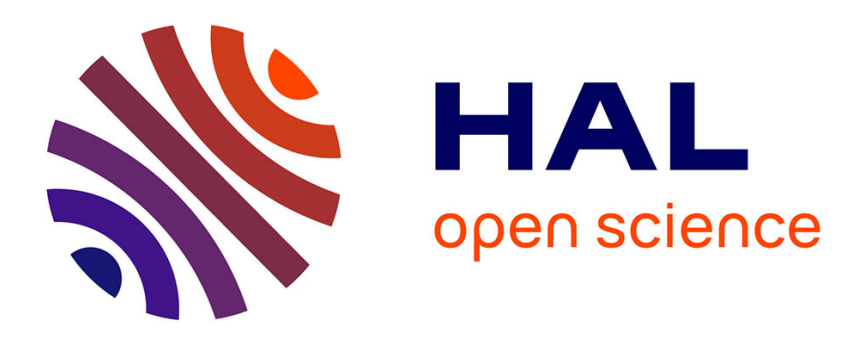

\title{
Assessment of Thermoset Cure-Induced Strains by Fiber Bragg Grating Sensor
}

\author{
Laurent Robert, Gilles Dusserre
}

\section{To cite this version:}

Laurent Robert, Gilles Dusserre. Assessment of Thermoset Cure-Induced Strains by Fiber Bragg Grating Sensor. Polymer Engineering and Science, 2014, 54 (7), p. 1585-1594. 10.1002/pen.23700 . hal-01611617

\section{HAL Id: hal-01611617 https://hal.science/hal-01611617}

Submitted on 8 Nov 2018

HAL is a multi-disciplinary open access archive for the deposit and dissemination of scientific research documents, whether they are published or not. The documents may come from teaching and research institutions in France or abroad, or from public or private research centers.
L'archive ouverte pluridisciplinaire HAL, est destinée au dépôt et à la diffusion de documents scientifiques de niveau recherche, publiés ou non, émanant des établissements d'enseignement et de recherche français ou étrangers, des laboratoires publics ou privés. 


\title{
Assessment of Thermoset Cure-Induced Strains by Fiber Bragg Grating Sensor
}

\author{
Laurent Robert, Gilles Dusserre \\ Université de Toulouse; Mines Albi, INSA, UPS, ISAE, ICA (Institut Clément Ader), \\ Campus Jarlard, F-81013 Albi Cedex 09, France
}

\begin{abstract}
Composites based on thermosetting resin and reinforcement fibers present generally unwanted residual internal stresses inherent to their elaboration process. In this article, different curing experiments of thermosets (isothermal and anisothermal) were monitored using optical fiber Bragg grating (FBG) sensors and thermocouples, to assess the cure-induced strains. A thermokinetic model evaluates the degree of conversion of the resin. At the onset of stress transfer to the optical fiber, the degree of conversion ranges between 0.63 and 0.68 . During curing, the FBG deforms under chemical shrinkage with an amplitude widely lower than the expected chemical strain, showing that the FBG signal is not directly related to the actual chemical shrinkage. However, once the resin is cured, the FBG sensor provides directly the coefficient of thermal expansion of the resin, as function of temperature and degree of conversion, reached in the different experiments.
\end{abstract}

\section{INTRODUCTION}

Fiber-reinforced composites are generally used as structural materials because of their excellent specific strength. However, they can suffer for sensitivity to damage and impact (delamination) [1], and their failure properties are not always well predicted. This is caused partially by residual internal stresses [2] and flaws, originating from the elaboration process (porosity, curing defect). The process can be monitored by including adequate high performance in-situ sensing systems [3], allowing a better knowledge of the process cycle and the related parameters.

An important issue that has promoted the development of in-situ composite process instrumentation is the knowledge of cure residual stresses (CRS) [2-4]. Indeed, process parameters have an important impact on the properties of the composite structure, and particularly in the development of CRS. CRS can cause important warpage, they can affect the final material properties and have an influence on the sensitivity to damage. The fine knowledge of the development, characterization, and amplitude of these CRS is thus necessary to reduce their effects thanks to an optimization of the cure cycle by adjusting the process parameters.

CRS have three main origins: chemical, thermal, and mold-laminate interaction origins. The chemical origin comes from the network structure of the thermoset which is built during polymerization. The reaction of reticulation and the formation of these networks induce volume variations of the matrix usually named chemical shrinkage. In a fiber-reinforced composite, fibers are opposed to the matrix chemical contraction, they are thus submitted to compressive stresses whereas the matrix is submitted to tensile stresses. During reticulation, at temperature above the glass transition, the viscosity of the network allows the relaxation of an important part of the internal stresses. Nevertheless, the level of chemical contraction of high performance epoxy systems remains relatively low, for example, volumetric shrinkage of RTM6 is approximately $2.7 \%$ [5]. However, very few studies report quantitative values of stress transfer onset on laminate fibers as well as chemical shrinkage level transferred to the fibers.

The CRS from thermal origin comes from the mismatch between the coefficients of thermal expansion (CTE) of all the involved components in presence, as manufacturing structural composites implies curing at a relatively high temperature (typically around $180^{\circ} \mathrm{C}$ ). At the scale of the components, differential CTE between fibers and matrix produces internal stresses in the composite material. At the scale of the laminate, CRS are induced because of the anisotropic character of plies in terms of expansion behavior, stresses appearing mainly during the cooling to room temperature.

The third cause of CRS is linked to interactions between mold and composite caused by their differential thermoelastic behaviors. Stresses due to differences of CTE between tools and reinforcement give rise to strain in the adjacent reinforcement ply and gradually to the other plies. 
From two decades, it has been reported the use of several optical fiber sensors for the measurement of residual strains in composite, most often extrinsic Fabry-Perot interferometric sensor [6] and gratings-based sensors [24, 7-9]. Optical fiber sensors offer good solutions to address measurements of internal strains because of their intrinsic advantages: they are small and flexible, light, immune to electromagnetic interference, and can withstand corrosive environments and high temperatures. One of the most interesting candidate is the fiber Bragg grating (FBG) sensor, which can provide localized measurements with high sensitivity and good linearity. Moreover, strain gradients can be measured using the optical low-coherence reflectometry technique [10].

An important point is to know how and when the resin can transfer a load to the reinforcement fibers, giving rise to the onset of CRS. For instance, it has been reported in $[11,12]$, using fiber pull-out tests, that the onset of gelation is the key point of the stress transfer between fibers and matrix. Using embedded optical fiber sensors during composite processing is a great advantage, because even if the diameter of the optical fiber $(125 \mu \mathrm{m})$ is larger than the reinforcement fibers, the matrix may behave in the same way in the vicinity of both reinforcement fibers and optical fibers. The latter is thus and in a similar way submitted to induced strains, and can give information about the beginning, the origin, and the intensity of the CRS.

In this article, both chemical and thermal cure-induced strains are investigated from the measurement of temperature (thermocouples), strain (FBG sensors), and degree of conversion (thermokinetic model). The occurrence of the stress transfer is highlighted during the cross-linking of a thermoset resin subjected to several thermal cycles, mainly isothermal reticulation, cooling at a fixed degree of conversion, and anisothermal reticulation. The next section describes the materials and methods used for this work. The results are shown in section RESULTS for isothermal and anisothermal curing experiments and discussed in the last section.

\section{EXPERIMENTAL METHODS AND MODELIZATION}

\section{FBG Sensor}

A FBG is a local permanent submicron period refractive index modulation in the core of an optical fiber, created by an appropriate sideways illumination with UVlight of a photosensitive optical fiber [13]. When light with a broadband spectrum travels into the optical fiber and interacts with the grating, it acts to couple the fundamental forward propagating mode to the contrapropagating core mode. This coupling occurs at the specific socalled Bragg wavelength $\lambda_{\mathrm{B}}$, with the FBG acting as a narrow-band reflection filter. The Bragg wavelength $\lambda_{\mathrm{B}}$ is defined by the grating period $\Lambda$ (typically $0.5 \mu \mathrm{m}$ ) and the effective refractive index of the propagating mode $n_{\text {eff }}\left(1.4682\right.$ for a Corning SMF-28 ${ }^{\mathrm{TM}}$ fiber at $\left.1550 \mathrm{~nm}\right)$, according to the well-known Bragg equation, Eq. 1 .

$$
\lambda_{\mathrm{B}}=2 n_{\mathrm{eff}} \Lambda
$$

A change in either one of these parameters changes the wavelength. A temperature increase causes a thermal expansion of the grating (and thus a change of its period) and also a change in the refractive index. A mechanical strain deforms the FBG and thus changes its period, and modifies also the refractive index by photoelastic effects.

In a general point of view, let us consider the case of a FBG embedded in a host material that submits it to a three-dimensional (3D) thermomechanical stress field. Under the assumptions of uniform strain field along the grating and negligible shear and bending, the strains of the fiber core are the same as the strains of the optical fiber. The relationship that expresses the wavelength shift versus the three principal strains $\varepsilon_{x}, \varepsilon_{y}, \varepsilon_{z}$ ( $z$ is the direction of the fiber axis) and the temperature change $\Delta T$ is as follows [14, 15]:

$$
\left\{\begin{array}{l}
\frac{\Delta \lambda_{\mathrm{B}, x}}{\lambda_{\mathrm{B}}^{0}}=\varepsilon_{z}^{*}-\frac{n_{\mathrm{eff}}^{2}}{2}\left[p_{11} \varepsilon_{x}^{*}+p_{12}\left(\varepsilon_{y}^{*}+\varepsilon_{z}^{*}\right)\right]+\mathrm{CTE} \cdot \Delta T+\xi \cdot \Delta T \\
\frac{\Delta \lambda_{\mathrm{B}, y}}{\lambda_{\mathrm{B}}^{0}}=\varepsilon_{z}^{*}-\frac{n_{\mathrm{eff}}^{2}}{2}\left[p_{11} \varepsilon_{y}^{*}+p_{12}\left(\varepsilon_{x}^{*}+\varepsilon_{z}^{*}\right)\right]+\mathrm{CTE} \cdot \Delta T+\xi \cdot \Delta T
\end{array}\right.
$$

where the strain components $\varepsilon_{x}, \varepsilon_{y}, \varepsilon_{z}$ are decomposed into a sum of the thermal expansion and the mechanical strains $\varepsilon_{x}^{*}, \varepsilon_{y}^{*}, \varepsilon_{z}^{*}$ induced by stress.

In $E q .2, \lambda_{\mathrm{B}}^{0}$ is the initial Bragg wavelength, $\mathrm{CTE}=\frac{1}{\Lambda} \frac{\partial \Lambda}{\partial T}$ is the coefficient of thermal expansion of the optical fiber $\left(0.55 \times 10^{-6} \mathrm{~K}^{-1}\right.$ for silica $), \xi=\frac{1}{n_{\mathrm{eff}}} \frac{\partial n_{\text {eff }}}{\partial T}$ represents the thermo-optic coefficient $\left(7.3 \times 10^{-6} \mathrm{~K}^{-1}\right.$ for SMF-28 ${ }^{\mathrm{TM}}$ silica-core fiber), and $p_{11}$ and $p_{12}$ are the Pockel's strain-optic coefficients $\left(p_{11}=0.113\right.$ and $\left.p_{12}=0.252[14]\right)$. Note that the index change is by far the dominant thermal effect. As shown by Eq. 2, due to the birefringence effect, the Bragg peak is split into two distinct ones. In the present study, we consider only an axisymmetric configuration and thus $\varepsilon_{r}=\varepsilon_{x}=\varepsilon_{y}$ is the radial strain, and $\varepsilon_{z}$ is the axial strain. In this case, both peaks are superimposed and the Bragg wavelength variation is given by $E q .3$ :

$\frac{\Delta \lambda_{\mathrm{B}}}{\lambda_{\mathrm{B}}^{0}}=\varepsilon_{z}^{*}\left\{1-\frac{n_{\mathrm{eff}}^{2}}{2}\left[p_{12}+\left(p_{11}+p_{12}\right) \frac{\varepsilon_{r}^{*}}{\varepsilon_{z}^{*}}\right]\right\}+\mathrm{CTE} \cdot \Delta T+\xi \cdot \Delta T$

The thermal contraction/dilatation of a host material in which the FBG is embedded, is here included in the mechanical strain $\varepsilon^{*}$ as it results in an occurrence of thermomechanical stresses. The information that is expected from the FBG measurement is the axial mechanical strain $\varepsilon_{z}^{*}$ that reveals the state of stress of the fiber in its 
TABLE 1. Material parameters of optical fiber SMF-28 ${ }^{\circledR}$ [14].

\begin{tabular}{lccc}
\hline Radius $[R(\mu \mathrm{m})]$ & $\begin{array}{c}\text { Elastic modulus } \\
{[E(\mathrm{Gpa})]}\end{array}$ & $\begin{array}{c}\text { Poisson's } \\
\text { ratio }(v)\end{array}$ & $\begin{array}{c}\text { Coefficient of thermal } \\
\text { expansion }\left[\mathrm{CTE}\left(\mathrm{K}^{-1}\right)\right]\end{array}$ \\
\hline 62.5 & 70 & 0.17 & $0.55 \times 10^{-6} \mathrm{~K}^{-1}$ \\
\hline
\end{tabular}

environment. It is therefore mandatory to know the temperature change $\Delta T$ and the biaxiality ratio defined as $B=\varepsilon_{r}^{*} / \varepsilon_{z}^{*}$.

A first issue appears when the two solicitations, mechanical strain and temperature change, coexist simultaneously. Many publications have been devoted to the discrimination of the temperature and strain responses of FBG sensors and a lot of techniques have been reported to overcome this problem $[13,16,17]$. The most classical extrinsic scheme consists of two FBGs, with one encapsulated to remain free from mechanical stresses [8]. Optical intrinsic schemes are also reported that ensure an optimum spatial resolution as the measurement is performed exactly at the same location, for example, by superimposing a FBG and a long-period grating [9, 18]. A thermocouple can also be associated to a FBG to collect the thermal component [4]. This last method was applied in the present study. From Eq. 3, a thermal calibration by keeping the FBG free from mechanical stresses allows to evaluate the thermal sensitivity $K_{\mathrm{T}}=\lambda_{\mathrm{B}}^{0}(\mathrm{CTE}+\xi) \approx 12$ pm $\mathrm{C}^{-1}$ at $1550 \mathrm{~nm}[13,18]$.

The second issue comes from the knowledge of the biaxiality ratio $B=\varepsilon_{r}^{*} / \varepsilon_{z}^{*}$, function of the thermomechanical loading, that can only be assessed by considering a particular configuration of the fiber embedded in the host material. In our case, we consider a host material (i.e., the epoxy resin) subjected to isotropic volume change due to chemical shrinkage and thermal contraction. An analytical model is proposed in the following to evaluate
$B$. This model involves an infinite straight optical fiber (radius $R$, material parameters: Young's modulus $E$, Poisson's ratio $v$, coefficient of thermal expansion CTE) embedded in an infinite coaxial resin cylinder (radius $R_{\mathrm{e}}$, material parameters: Young's modulus $E^{r}$, Poisson's ratio $v^{r}, \mathrm{CTE}^{r}$ ). The problem is axisymmetric and invariant by translation along the fiber axis $z$. The momentum balance of the system subjected to a temperature change is solved by assuming the continuity of the displacements and of the radial stress at the interface between the two materials, and a free external boundary of the resin. Results allow to calculate the radial strain $\varepsilon_{r}$ and the axial strain $\varepsilon_{z}$ function of the temperature change $\Delta T$, of the resin cylinder $R_{\mathrm{e}}$ and of the resin parameters $E^{r}, v^{r}$ and $\mathrm{CTE}^{r}$, the parameters relative to the optical fiber being known and given in Table 1.

The first remark is that the biaxiality ratio $B=\varepsilon_{r}^{*} / \varepsilon_{z}^{*}$ is independent of the temperature change because each strain component is proportional to $\Delta T$. It is interesting to analyze the change in $B$ by varying the elastic modulus of the resin and the external radius of the resin cylinder. The other parameters $v^{r}$ and $\mathrm{CTE}^{r}$ are assumed to be constant and set, respectively, to 0.4 and $60 \times 10^{-6} \mathrm{~K}^{-1}$ (see section Curing Cycles) corresponding to the particular case of the cooling of a vitrified resin at the end of the curing process. Results are shown in Fig. 1a for $E^{r}$ in the range [0.1-10] $\mathrm{GPa}$ and $R_{\mathrm{e}}$ in the range [62.5-1000] $\mu \mathrm{m}$.

As expected, for an infinite amount of resin surrounding the optical fiber and an infinite small elastic modulus, $B$ tends toward $-v$. In this particular case, Eq. 3 can be simplified and gives the strain sensitivity of a naked $\mathrm{FBG}, K_{\varepsilon} \approx 1.23 \mathrm{pm}(\mu \varepsilon)^{-1}$ at $1550 \mathrm{~nm}[13,18]$. In all other cases, $B$ should be evaluated correctly knowing the actual values of the parameters of the surrounding host material. On the one hand, it remains very complex to

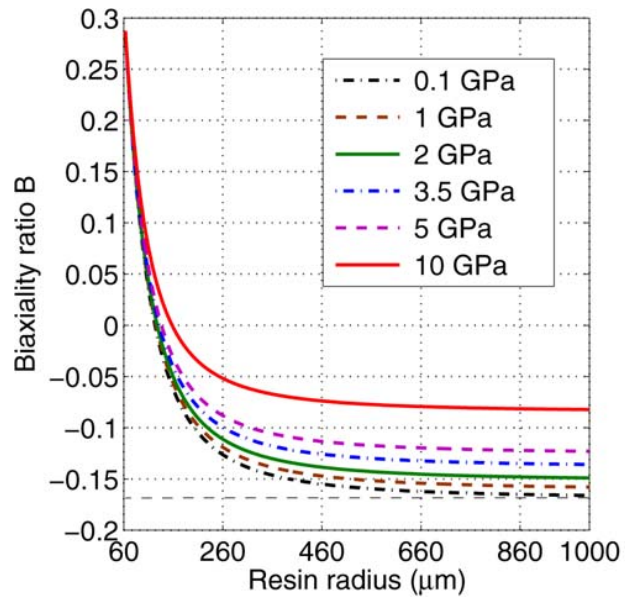

(a)

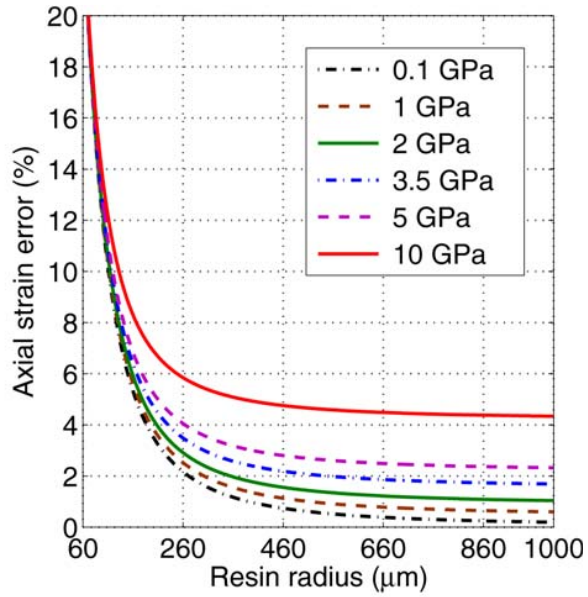

(b)

FIG. 1. (a) Biaxiality ratio and (b) axial strain error committed by assuming a constant $B$ ratio, function of $R_{\mathrm{e}}$ for various resin elastic modulus. [Color figure can be viewed in the online issue, which is available at wileyonlinelibrary.com.] 
process the FBG signal by taking into account the variation of $B$ during the experiment using the results of a thermomechanical modeling. On the other hand, it is very easy to calculate the axial strain error committed when assuming a constant $B$ ratio. The axial error $\operatorname{err}(B)$ plotted in Fig. 1b function of $R_{\mathrm{e}}$ for various resin elastic modulus is defined by Eq. 4 .

$$
\operatorname{err}(B)=\frac{\varepsilon_{z}^{*}(B)-\varepsilon_{z}^{*}(v)}{\varepsilon_{z}^{*}(B)} * 100
$$

The actual configuration of the experiments involves a $1-\mathrm{mm}$ resin radius (thickness of the sample higher than 2 $\mathrm{mm}$ ), and a resin Young's modulus $E^{r}$ varying between 0 and $3.5 \mathrm{GPa}$ along with the polymerization. The value of $B$ may thus vary between -0.13 and -0.17 for the whole set of experimental data under study (Fig. 1a). It was chosen to consider $B=-0.17$ (fiber Poisson's ratio) that leads to a negligible error when the resin modulus is close to zero. The maximal error in the measured axial strain is lower than $2 \%$ (Fig. 1b) and is obtained when the resin is fully cured. Note that this error level is acceptable in many cases (typically for isotropic surrounding material with relatively low modulus compared to the one of the optical fiber), but in some cases considering $B$ as the optical fiber Poisson's ratio can induce very erroneous data for instance when the host material is anisotropic as in the case of a composite material [19].

\section{Materials and Experimental Methods}

The resin under study is the HexFlow ${ }^{\circledR}$ RTM6 from Hexcel $^{\circledR}$ Composites. RTM6 is a premixed epoxy resin specifically dedicated to liquid composite molding and commonly used industrially to manufacture aeronautic carbon-reinforced composite parts.

Three kinds of mold were used to analyze the effect of the mold interaction on the resin cure-induced strain as well as to enhance or not the exothermicity of the reaction. A rigid metallic $(\mathrm{RM})$ mold, which consists basically of a $0.9-\mathrm{mm}$ thick $120 \times 60 \mathrm{~mm}^{2}$ steel plate, was used as a standard material for mold. It was treated with a mold release agent (Loctite ${ }^{\circledR}$ Frekote $^{\circledR}$ ). A soft metallic (SM) mold was made of a simple $0.05-\mathrm{mm}$ thick circular $(\sim 80$ $\mathrm{mm}$ diameter) aluminum foil pan, thus easily deformable, and enabling large thermal transfer. The thickness of the resin samples was, respectively, of 2.85 and $2.4 \mathrm{~mm}$ in these two first molds. The last mold was a 1-mm thick silicon (SI) circular ( $\sim 80$-mm diameter) mold that exhibited high thermal insulation. This mold was used to enhance the exothermicity of the reaction and to ensure an anisothermal curing. In this case, the thickness of the resin sample was $12.8 \mathrm{~mm}$. These molds were simply placed on a steel plate in a thermally controlled oven. Type $\mathrm{K}$ microthermocouples $(110-\mu \mathrm{m}$ diameter) were used with a Pico ${ }^{\circledR}$ TC-08 controlled device to assess the temperature in the resin and the oven throughout the curing cycles.

The FBGs used in this work are commercially available apodized 5-mm length Bragg gratings from Welltech instrument Company Limited (Hong Kong), written on bare (uncoated) SMF-28 ${ }^{\circledR}$ Corning ${ }^{\circledR}$ optical fibers. Before embedment, each FBG was stabilized by heat treatment at $180^{\circ} \mathrm{C}$ for $3 \mathrm{~h}$. The $\mathrm{FBG}$ were simply placed in each mold at the symmetry center of the specimens (as the thermocouples), in the middle of the resin thickness. Bragg wavelength shifts were measured with a Micron Optics si425 optical sensing interrogator (wavelength resolution $2.5 \mathrm{pm}$, accuracy $1 \mathrm{pm}$, and repeatability within $0.5 \mathrm{pm})$.

\section{Cure Modeling}

To study the signals provided by the FBG sensors, a thermokinetic model is used to assess the degree of conversion and the glass transition temperature against time. This section describes briefly this model that has been implemented for each experiment. This approach was detailed and experimentally validated in a previous paper [20] by comparison to dielectric analysis and Fresnel refractometer measurements. This model solves the heattransfer equation including a heat source term resulting from the polymerization, and calculated by a thermokinetic model (Kamal-Sourour modified to take into account diffusion mechanisms below the glass transition temperature). The variation of glass transition temperature is described by a Di-Benedetto law. All the coefficients used in this model come from the literature (see [20-25] for details) as well as the different thermal properties of RTM6.

Two different resolution schemes are used. For thin resin samples (SM and RM experiments), it was demonstrated that an assumption of uniform temperature in the thickness is relevant. A semianalytical resolution based on a finite differences integration scheme is thus possible, which can easily be coupled to an optimization algorithm. For thick resin samples (SI experiment), significant thermal gradients require a 2D finite element resolution (the commercial software COMSOL Multiphysics ${ }^{\circledR}$ was used).

Whatever the resolution method, the model must be adjusted by identifying the heat exchange coefficient. An optimization allows to find the value of the heat exchange coefficient between the resin and its environment that minimizes the gap between the computed and measured resin temperature (the temperature in the oven and in the resin sample are recorded during each experiment). Excellent results are obtained for isothermal and anisothermal curing, as shown in section Strain Induced during the Curing, where the calculated resin temperature perfectly fits the experimental one. In some cases, the identification requires also to evaluate the initial degree of conversion of the resin, when setting it to zero provides time shifted results. All these developments are discussed in [20]. 


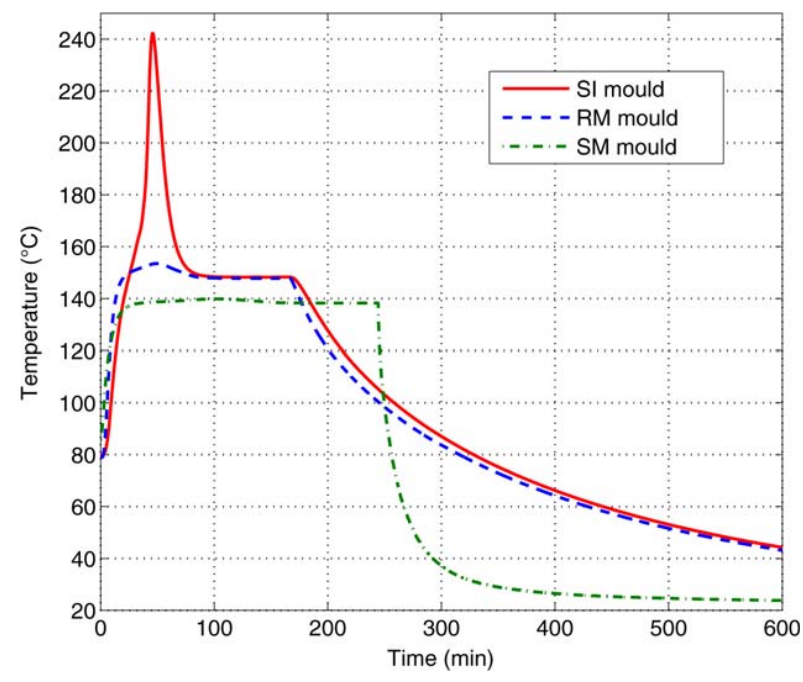

FIG. 2. Temperature variation throughout the curing cycle of the resin for the three involved molds. [Color figure can be viewed in the online issue, which is available at wileyonlinelibrary.com.]

The last additional information that is needed in this study is the chemical strain $\varepsilon_{\text {chem }}$ of the resin. This data are provided by Aduriz et al. [24] who performed shrinkage experiments of RTM6 at $150^{\circ} \mathrm{C}$, and report a linear relationship between the specific volume and the degree of conversion.

\section{RESULTS}

This section is devoted to the presentation of the main results. First, the whole curing cycles are presented and global residual strains are analyzed. Then, the cureinduced strains deduced from the FBGs are systematically and quantitatively investigated along with the degree of conversion of the resin, for the different experiments.

\section{Curing Cycles}

Several curing experiences were conducted in the different molds cited above (RM, SM, and SI). In each case, a FBG sensor together with a thermocouple was inserted into the mold to monitor the curing reaction. The thermocouple was also used in the processing of the FBG signal to discriminate strain effects from thermal effects according to $E q$. 3. Figure 2 presents the temperature change with time for the three curing experiments, whereas Fig. 3 depicts the corresponding Bragg wavelength shifts.

The thermal cycle consists of an increase in temperature from $80^{\circ} \mathrm{C}$ at a heating rate of $6^{\circ} \mathrm{C} \min ^{-1}$ to limit the temperature gradients (heating phase), followed by a cure plateau where the temperature setting stands at $140^{\circ} \mathrm{C}$ for $200 \mathrm{~min}$ for the SM mold, and $150^{\circ} \mathrm{C}$ for 130 min for the SI and RM molds. The third period is a simple cool down by natural convection to ambient temperature (cooling phase). Note that for the SM experiment the convection was enforced.

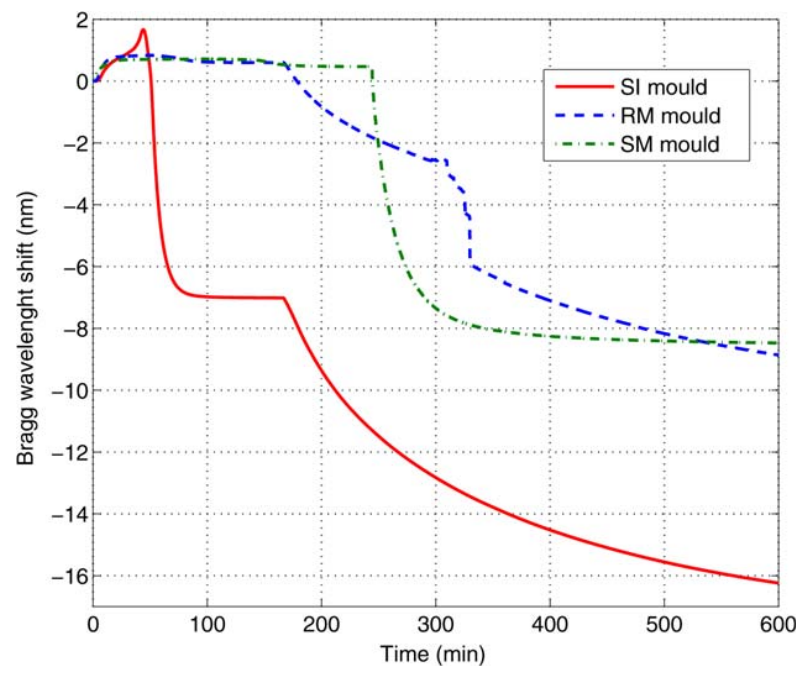

FIG. 3. Bragg wavelength shift throughout the curing cycle of the resin for the three involved molds. [Color figure can be viewed in the online issue, which is available at wileyonlinelibrary.com.]

During the cure plateau, the behavior depends on the type of mold. For the SI mold, it is observed in Fig. 2 a huge thermal overshoot which corresponds to the exothermal manifestation of chemical bonding: the temperature reaches more than $240^{\circ} \mathrm{C}$ as a result of a thick resin sample and an insulating mold. For the RM mold, a small exothermal effect is seen. For the SM mold, the exothermal effect is nearly not detectable because the temperature setting was lowered by $10^{\circ} \mathrm{C}$.

A first rough analysis of the Bragg wavelength variations during the experiments (see Fig. 3) allows us to make the following comments.

First, a small wavelength increase occurs, corresponding to the temperature increase of the liquid state resin. Considering the SI experiment, after the temperature overshoot, a large decrease in wavelength is observed at the same time as the decrease in temperature after the exothermal peak. For both metallic molds experiments, no significant wavelength variation occurs during the cure plateau. At the end of the cure plateau, a large decrease in wavelength is observed, corresponding to the thermal contraction of the cured resin in a solid state. Note that in the RM mold the cooling phase shown in Fig. 3 is separated in two parts. This is explained by a debonding of the cured resin from the metallic plate, and also illustrated in Fig. 4. For this figure, the mechanical strain experienced by the FBG sensors immerged in the resin during the cure cycles was calculated from Eq. 3 using the measured Bragg wavelength variations together with their associated temperature variations measured in each mold.

The thermal sensitivities were estimated from temperature and wavelength shift during the heating phase of each experiment (see Fig. 2 and 3), under the assumption that FBGs are free of mechanical strain in a liquid 


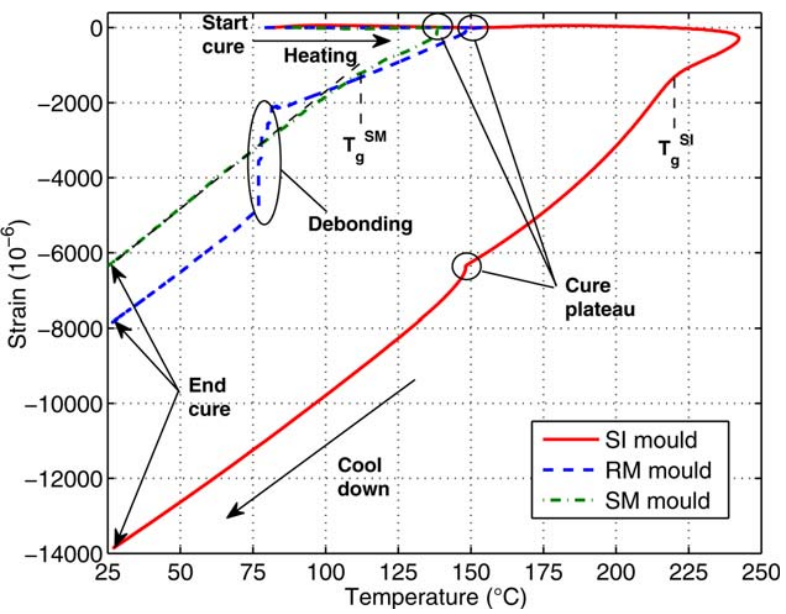

FIG. 4. Mechanical strain variations versus temperature throughout the curing cycle of the resin for the three involved molds. [Color figure can be viewed in the online issue, which is available at wileyonlinelibrary. com.]

surrounding material. Quite usual values were found, respectively, $11.25,11.4$, and $11.45 \mathrm{pm} \mathrm{K}^{-1}$ for the FBG located into the RM, the SM, and the SI molds. In Fig. 4, it is verified that all curves are superimposed during the heating phase and an almost zero strain is measured, due to the fluid state of the resin whatever the experiment.

The large exothermal peak is clearly emphasized for the SI experiment. Before the temperature reaches its maximum (about $225^{\circ} \mathrm{C}$ ), the strain begins to decrease as the temperature increases. This shows that stress transfer has occurred, and at that time the measured strains originate from a chemical shrinkage. After the exothermal peak, an inflexion point corresponding to the glass transition temperature $T_{\mathrm{g}} \mathrm{SI}$ is highlighted, and will be discussed further. The cure plateaus are shown by the circles in Fig. 4. The strains developed during this phase seem particularly small. During the cooling phase, the behavior depends on the considered mold. It is supposed here that after the cure plateau, a certain degree of conversion is obtained in reference to the curing temperature reached, and therefore that the material undergoes only contraction from thermal origin. This will be confirmed further.

For the RM mold, the cool down is divided into three steps: (i) the strain first decreases linearly with temperature, leading to what is usually considered in the literature as an apparent CTE of the twin-material of $30.9 \mu \varepsilon \mathrm{K}^{-1}$. In this case, the thermal contraction of the resin is hindered by the lower thermal contraction of the metallic mold. (ii) A sudden jump of $3000 \mu \varepsilon$ occurs because the resin debonds from the RM mold. (iii) From the jump to the end of the cooling, the slope of the line in Fig. 3 (apparent CTE of the cured resin) is found to be $58.1 \mu \varepsilon$ $\mathrm{K}^{-1}$, as it is considered that the mold does not constrain the cured resin.

For the SM mold, a careful observation of Fig. 4 reveals a nonlinear variation of the mechanical strain after reticulation. At the beginning of the cool down, the
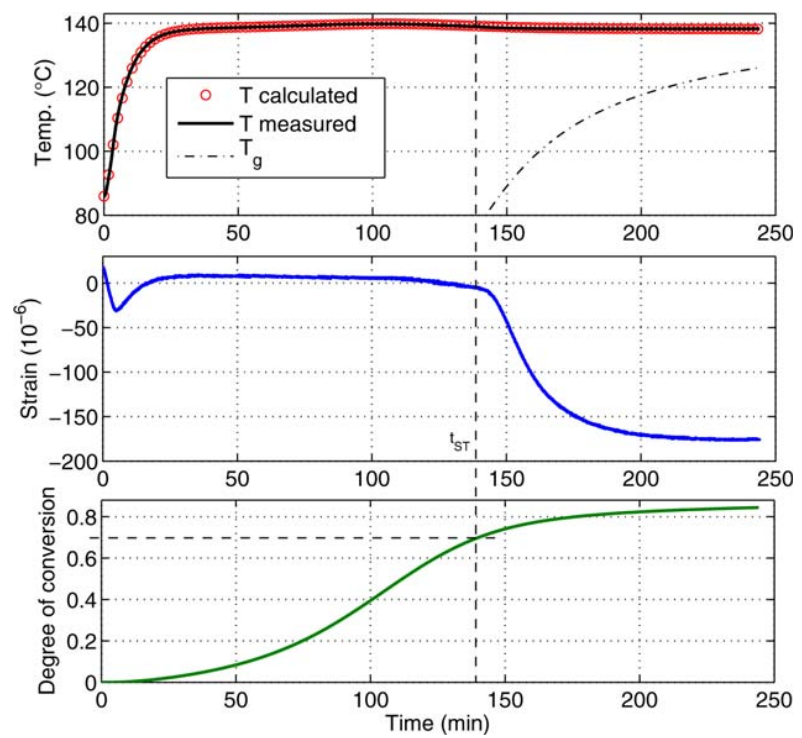

FIG. 5. Temperature, mechanical strain, and degree of conversion versus time for the temperature increase and the cure plateau at $138^{\circ} \mathrm{C}$ of the RTM6 curing cycle in the SM mold. [Color figure can be viewed in the online issue, which is available at wileyonlinelibrary.com.]

temperature is above the glass transition temperature $T_{g}$ of the reticulated resin. Around $110-120^{\circ} \mathrm{C}$, the temperature crosses down $T_{\mathrm{g}} \mathrm{SM}$ and the resin becomes glassy, resulting in a change in the apparent CTE. Below $100^{\circ} \mathrm{C}$ when the strain varies linearly with the temperature, the apparent CTE of the cured resin can be estimated by the slope of the straight line portion of the curve at $61.7 \mu \varepsilon \mathrm{K}^{-1}$.

For the SI mold allowing totally free deformations of the resin during the whole curing cycle, from the end of the cure plateau at $150^{\circ} \mathrm{C}$ to the cooling end, the total strain shifts also linearly with temperature. In this case, the apparent CTE of the cured resin is estimated at 56.6 $\mu \varepsilon \mathrm{K}^{-1}$, a value of the same order as the other values found.

\section{Strain Induced During the Curing}

In this section, are detailed the measured strains from the FBG signal together with the degree of conversion $\alpha$ of the resin resulting from the aforementioned thermokinetic cure model. For the three experiments, the models were calibrated as previously explained [20]. The results fit well the measured temperature cycle, showing that the models are able to physically represent both isothermal as anisothermal curing. It allows to give an estimation of the degree of conversion and also of the glass transition temperature $T_{\mathrm{g}}$. The chemical strain $\varepsilon_{\text {chem }}$ is deduced from the degree of conversion $\alpha$ using the change in the specific volume with $\alpha$ provided by the result of an experiment at $150^{\circ} \mathrm{C}$ reported in [24].

Figure 5 presents the change in temperature, mechanical strain, and degree of conversion with time for the heating phase and the cure plateau, in the SM mold. 

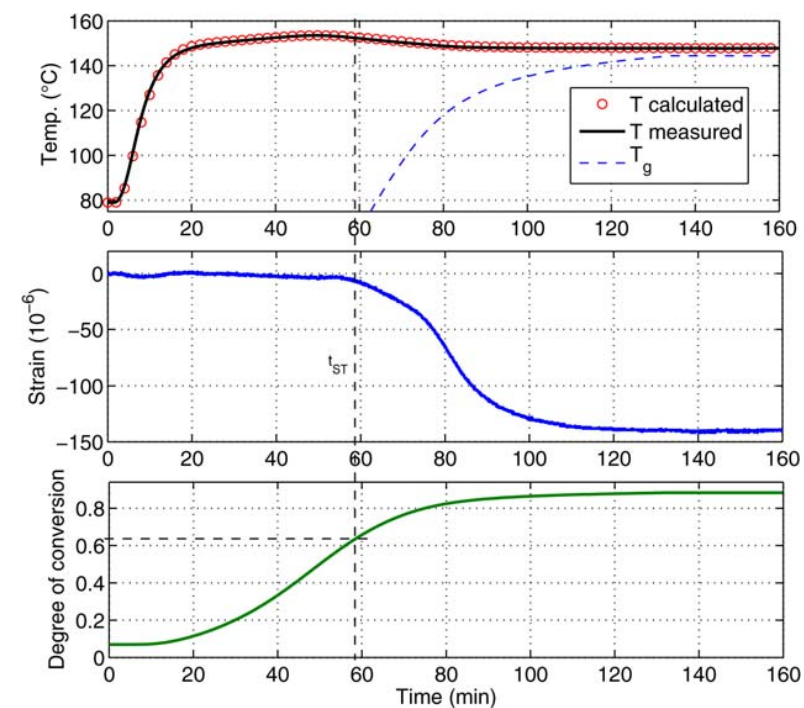

FIG. 6. Temperature, mechanical strain, and degree of conversion versus time for the temperature increase and the cure plateau at $148^{\circ} \mathrm{C}$ of the RTM6 curing cycle in the RM mold. [Color figure can be viewed in the online issue, which is available at wileyonlinelibrary.com.]

During the heating phase, the small change in strain is due to the difference in the positioning of both sensors, and the presence of temperature gradients. The strain remains almost zero while the degree of conversion increases up to $\alpha\left(t_{\mathrm{ST}}\right) \approx 0.68$ (ST stands for stress transfer), see Fig. 5. From $t_{\mathrm{ST}}$, temperature remains constant but the strain (only due to chemical contraction) decreases up to $-175 \mu \varepsilon$ while $\alpha$ reaches it maximum value, about 0.846. Two main results are highlighted: the stress transfer to the fiber occurs for a fairly advanced level of polymerization, and the value of $\varepsilon_{\text {chem }}$ experienced by the FBG is well below that expected. In fact, it is deduced from [24] and the estimated degree of conversion, a

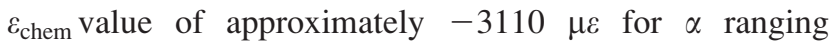
between 0.68 and 0.84 , significantly greater than that experienced by the FBG. Finally, the glass transition temperature given by the model is about $125^{\circ} \mathrm{C}$ at the end of the reaction. This value is very similar to the one estimated by the change in the slope of the cooling curve in Fig. 4 ( $T_{\mathrm{g}}^{\mathrm{SM}}$, section Curing Cycles).

Let us now turn to analyze the curing cycle of the resin inside the RM mold. Figure 6 illustrates the change in temperature, mechanical strain, and degree of conversion with time for the heating phase and the cure plateau. In the present experiment, the initial degree of conversion has been identified to $6.5 \%$ for a perfect match between experimental and calculated temperatures. The main tendencies are the same as previously observed for the SM mold: during the heating phase and a part of the cure plateau, the strain remains almost equal to zero while the degree of conversion increases up to $\alpha\left(t_{\mathrm{ST}}\right)$, estimated in this experience at about 0.63 . From $t=t_{\mathrm{ST}}$ to $t=100$ min, the strain evolves from 0 to $-140 \mu \varepsilon$ while $\alpha$ evolves from 0.63 to 0.884 . The stress transfer to the
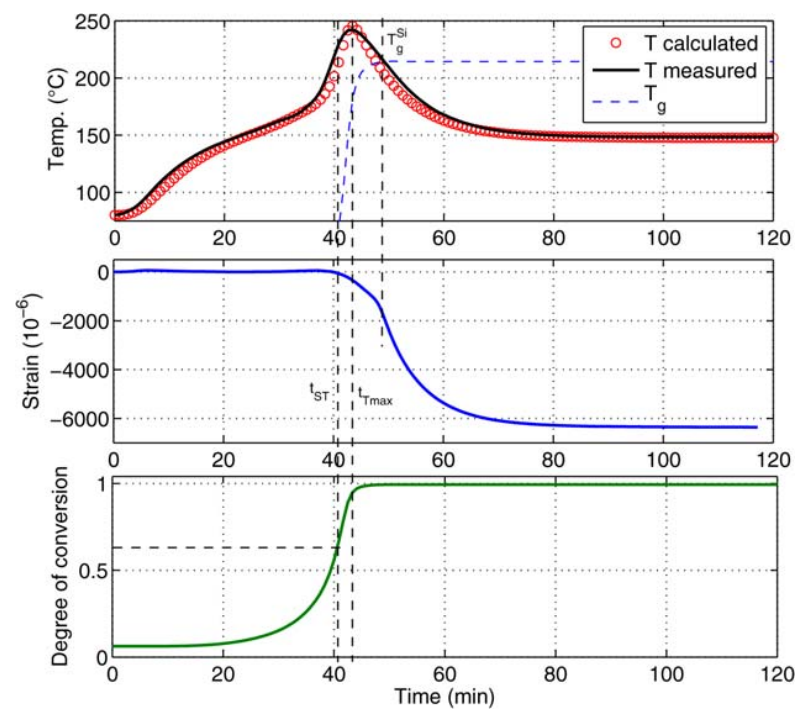

FIG. 7. Temperature, mechanical strain, and degree of conversion versus time for the temperature increase and the cure plateau at $148^{\circ} \mathrm{C}$ of the RTM6 curing cycle in the SI mold. [Color figure can be viewed in the online issue, which is available at wileyonlinelibrary.com.]

fiber occurs for a similar degree of conversion as in SM experiment, and the chemical strain measured by the FBG is also well below that expected, estimated at $-4770 \mu \varepsilon$ from [24] for this range of degree of conversion. Finally, the model shows that $T_{\mathrm{g}}$ is close to the curing temperature, this is why we do not detect any slope change at the beginning of the cooling curve in the RM mold.

Finally, the curing inside the SI mold is presented. The initial degree of conversion was also set to $6.5 \%$ for a perfect match between experimental and calculated temperatures [20]. Figure 7 shows the change in temperature, mechanical strain, and degree of conversion with time for the heating phase, temperature overshoot, and cure plateau that occurred in the SI mold. The strain remains zero until $t_{\mathrm{ST}}$ and $\alpha\left(t_{\mathrm{ST}}\right) \approx 0.66$. From $t_{\mathrm{ST}}$ to $t_{\mathrm{Tmax}}$ corresponding to the maximum temperature of the exothermal peak, where $\alpha\left(t_{\text {Tmax }}\right) \approx 0.95$, a decrease of the strain of about $-300 \mu \varepsilon$ is measured, but the increase in the temperature (about $25^{\circ} \mathrm{C}$ ) implies an underestimation of the measurement of this chemical strain by the FBG. The stress transfer to the fiber occurs for a similar degree of conversion as previously and the chemical strain measured by the FBG is also well below that expected, -6100 $\mu \varepsilon$, estimated from [24] for this range of degree of conversion. From $t_{\text {Tmax }}$ to $t=80 \mathrm{~min}$, the temperature decreases strongly and results in a large decrease in strain (about $-6360 \mu \varepsilon$ ) mainly conducted by the thermal strain because $\alpha$ changes only from 0.95 to 1 . During this cooling, the temperature of the resin crosses the glass transition temperature $T_{\mathrm{g}}^{\mathrm{SI}}$, and an inflexion point is seen in Fig. 6 on the strain curve as well as in Fig. 3. At the end of this last experiment, the resin is fully cured and vitrified. 


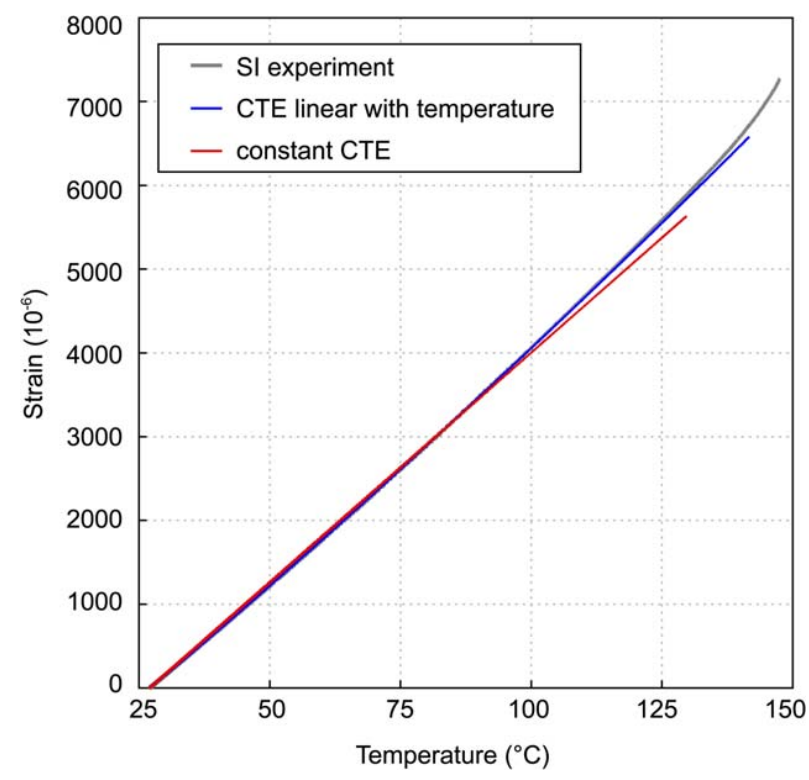

FIG. 8. Comparison of the thermal strain of fully cured RTM6 assuming a linear evolution of the CTE with temperature and a constant CTE value to the experimental data from SI experiment. [Color figure can be viewed in the online issue, which is available at wileyonlinelibrary. com.]

\section{DISCUSSION}

\section{Quasistatic Momentum Balance}

Let us consider an infinite unidirectional medium made of three components: a mold, a fiber, and a resin sample. The quantities related to each component are denoted by a superscript, respectively, $m, f$, and $r$. The different cross-sections are, respectively, $S^{m}, S^{f}$, and $S^{r}$.

The total strain rate of the medium is assumed to be uniform in each material and noted $\dot{\varepsilon}$. Thus, no warpage occurs. The total strain rate is decomposed into a mechanical part, a thermal part for each material, and a supplementary chemical part for the resin, Eq. 5.

$$
\dot{\varepsilon}=\left\{\begin{array}{l}
\dot{\varepsilon}^{m}=\dot{\varepsilon}_{\text {mech }}^{m}+\dot{\varepsilon}_{\text {therm }}^{m} \\
\dot{\varepsilon}^{f}=\dot{\varepsilon}_{\text {mech }}^{f}+\dot{\varepsilon}_{\text {therm }}^{f} \\
\dot{\varepsilon}^{r}=\dot{\varepsilon}_{\text {mech }}^{r}+\dot{\varepsilon}_{\text {therm }}^{r}+\dot{\varepsilon}_{\text {chem }}^{r}
\end{array}\right.
$$

The momentum balance inside the medium implies $E q$. 6. The three materials are supposed to behave as linear elastic bodies, the Young's modulus $E^{r}$ of the resin evolves with time because of reticulation and temperature changes, whereas the ones of the fiber, $E^{f}$, and the mold, $E^{m}$, are supposed to be constant $(E q .7)$. For the sake of simplicity, the viscous behavior of the resin above its glass transition is not accounted for what is obviously a strong assumption.

$$
S^{m} \dot{\sigma}^{m}+S^{f} \dot{\sigma}^{f}+S^{r} \dot{\sigma}^{r}=0
$$

$$
\left\{\begin{array}{l}
\dot{\sigma}^{m}=E^{m} \dot{\varepsilon}_{\text {mech }}^{m} \\
\dot{\sigma}^{f}=E^{f} \dot{\varepsilon}_{\text {mech }}^{f} \\
\dot{\sigma}^{r}=E^{r} \dot{\varepsilon}_{\text {mech }}^{r}+\dot{E}^{r} \varepsilon_{\text {mech }}^{r}
\end{array}\right.
$$

Using Eq. 7, the integration of Eq. 6 between two arbitrary instants $t_{0}$ and $t_{1}$ leads to $E q .8$.

$$
\begin{aligned}
& S^{m} E^{m}\left(\varepsilon_{\text {mech }}^{m}\left(t_{1}\right)-\varepsilon_{\text {mech }}^{m}\left(t_{0}\right)\right)+S^{f} E^{f}\left(\varepsilon_{\text {mech }}^{f}\left(t_{1}\right)-\varepsilon_{\text {mech }}^{f}\left(t_{0}\right)\right) \\
& +S^{r}\left[E^{r} \varepsilon_{\text {mech }}^{r}\right]_{t_{0}}^{t_{1}}=0
\end{aligned}
$$

If $t_{0}$ is chosen as the onset of stress transfer from resin to fiber (which can be the gel time, as reported in [11, $12]$ ), the mechanical strain of the fiber is then zero up to $t_{0}$.

Using Eq. 5 and assuming a constant coefficient of thermal expansion for the mold and the fiber, Eq. 8 can be rewritten as Eq. 9 .

$$
\begin{aligned}
& \left(S^{m} E^{m}+S^{f} E^{f}\right)\left(\varepsilon^{t_{1}}-\varepsilon^{t_{0}}\right)-\left(S^{m} E^{m} C T E^{m}+S^{f} E^{f} C T E^{f}\right)\left(T_{1}-T_{0}\right) \\
& +S^{r}\left(E^{r, t_{1}} \varepsilon_{\text {mech }}^{r, t_{1}}-E^{r, t_{0}} \varepsilon_{\text {mech }}^{r, t_{0}}\right)=0
\end{aligned}
$$

Using $E q .5$ and taking the temperature $T_{0}$, at time $t_{0}$, as the reference temperature for the thermal dilatation, Eq. 9 gives Eq. 10, which shows the complex interaction involved in the present case between the different materials.

$$
\begin{aligned}
\left(S^{m} E^{m}+\right. & \left.S^{f} E^{f}\right)\left(\varepsilon^{t_{1}}-\varepsilon^{t_{0}}\right)-\left(S^{m} E^{m} \mathrm{CTE}^{m}+S^{f} E^{f} \mathrm{CTE}^{f}\right)\left(T_{1}-T_{0}\right) \\
& +S^{r}\left(E^{r, t_{1}}\left(\varepsilon^{t_{1}}-\int_{t_{0}}^{t_{1}} \mathrm{CTE}^{r}(T, \alpha) \frac{d T}{d t} d t-\varepsilon_{\text {chem }}^{r, t_{1}}\right)\right. \\
& \left.-E^{r, t_{0}}\left(\varepsilon^{t_{0}}-\varepsilon_{\text {chem }}^{r, t_{0}}\right)\right)=0
\end{aligned}
$$

Obviously, a key point arises from the fact that both the CTE and the Young's modulus of the resin are functions of temperature and degree of conversion. Two particular cases are discussed further: cooling once the reticulation has ended (SI experiment) and isothermal cure (RM and SM experiments).

\section{Coefficient of Thermal Expansion of RTM6 at the End of the Experiments}

During the cooling to room temperature, and as discussed previously in section Curing Cycling, the effect of the mold is negligible in SM and SI experiments, as well as in RM experiment once debonding has occurred. In addition, the modulus of the resin for $T$ significantly lower than $T_{\mathrm{g}}$ can be assumed to be almost constant as it only depends on temperature once the reticulation has 


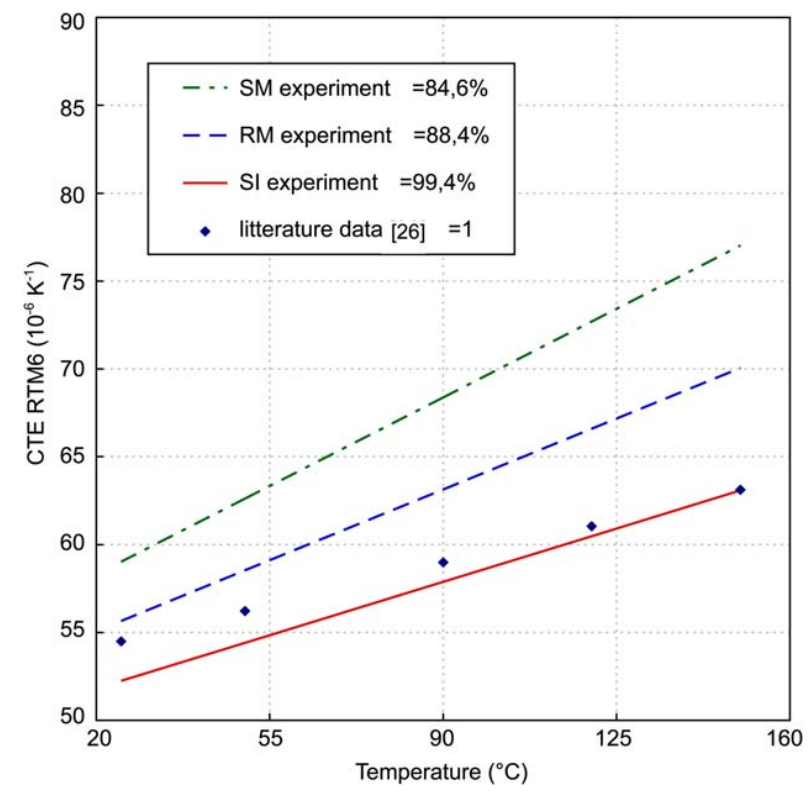

FIG. 9. CTE of RTM6 as a function of the temperature evaluated at the end of the SI, RM, and SM experiments compared to the experimental data reported by Hobbiebrunken et al. [26]. [Color figure can be viewed in the online issue, which is available at wileyonlinelibrary. com.]

ended (from $2.76 \mathrm{GPa}$ at room temperature to $1.9 \mathrm{GPa}$ at $150^{\circ} \mathrm{C}$ [26]). Finally, at the end of the experiments, $S^{f} E^{f}$ can be neglected compared to $S^{r} E^{r}$ (and not only $S^{f}<<$ $S^{r}$ ). In this particular case, the strain measured by the FBG is directly related to the thermal contraction of the resin (Eq. 11).

$$
\varepsilon^{t_{1}}-\varepsilon^{t_{0}}=\int_{t_{0}}^{t_{1}} \operatorname{CTE}^{r}\left(T, \alpha_{\text {end }}\right) \frac{d T}{d t} d t
$$

Hobbiebrunken et al. [26] reported a linear dependency of the CTE of RTM6 to temperature for a fully cured resin. Assuming this linearity, the evolution of the resin CTE with respect to temperature is identified using the FBG data at the end of the SI experiment. The time $t_{0}$ is taken at the end of the experiment. Figure 8 shows that this assumption allows to perfectly fit the experimental data from the FBG, compared to the assumption of a constant CTE. The linear evolution with temperature of the CTE of fully cured RTM6, identified from the SI experiment data, is compared to the experimental data reported by Hobbiebrunken et al. [26], Fig. 9. Despite a few overestimated slope of the curve, our results are very close to those of reference 26], with an error lower than 5\% between 25 and $150^{\circ} \mathrm{C}$.

The same identification was carried out for the SM and RM experiment, showing a variation of the CTE of the resin as a function of the degree of conversion of the resin. It is demonstrated that in-situ measurement using FBG sensor can be processed to assess the CTE of vitrified thermosets for various degree of cure.

\section{Isothermal Chemical Shrinkage Monitoring}

In an isothermal case, Eq. 10 can be reduced to Eq. 12 .

$$
\begin{aligned}
& \left(S^{m} E^{m}+S^{f} E^{f}\right)\left(\varepsilon^{t_{1}}-\varepsilon^{t_{0}}\right)+S^{r}\left(E^{r, t_{1}}\left(\varepsilon^{t_{1}}-\varepsilon_{\text {chem }}^{r, t_{1}}\right)\right. \\
& \left.-E^{r, t_{0}}\left(\varepsilon^{t_{0}}-\varepsilon_{\text {chem }}^{r, t_{0}}\right)\right)=0
\end{aligned}
$$

In Eq. 12 , the strain variation $\varepsilon^{t_{1}}-\varepsilon^{t_{0}}$ is given by the FBG and $\varepsilon_{\text {chem }}^{r, t_{1}}-\varepsilon_{\text {chem }}^{r, t_{0}}$ can be evaluated by the model using shrinkage data from the literature [24]. The former is one order of magnitude lower than the latter in both RM and SM experiments, see section Strain Induced during the Curing. This difference can be attributed on the one hand to the mold stiffness, and on the other hand, to the resin modulus variation, which remains the main unknown. In both experiments, the cure-induced strain of the fiber is almost the same, as well as the actual chemical shrinkage, despite a significant difference in the mold contribution $\left(S^{m} E^{m} / S^{r}\right.$ is 60 times higher in the RM mold). This assertion results from the underlying assumptions leading to $E q$. 10, that does not take into account the viscous relaxation in the resin. The mold does not interact with the resin in the vicinity of the fiber. As shown previously in section Coefficient of Thermal Expansion of RTM6 at the End of the Experiments, the assumption of Eq. 5, uniform strain in the fiber, the mold, and the resin, was valid for the fully cured resin. It is no longer valid here because the resin is not a solid, changing from a viscous liquid to a viscoelastic gel.

\section{SUMMARY AND CONCLUSIONS}

Curing experiments of RTM6 resin were conducted in several types of molds, each one instrumented with FBG and thermocouple, to assess the cure-induced strains. A thermokinetic model allowed to evaluate the degree of conversion of the resin throughout the cure. At the onset of stress transfer to the optical fiber, values of the degree of conversion between 0.63 and 0.68 are reported. This is slightly higher than the degree of conversion at gel point at $150^{\circ} \mathrm{C}$ reported in [24], that is, 0.59 , despite very different experimental approaches. Nevertheless, this seems logical as the resin must be rigid enough to deform the optical fiber, what is not the case just after the gel point where the Young's modulus is close to zero. Whatever the curing experiment, the FBG deforms under the chemical shrinkage of the resin. Nevertheless, the amplitude of the FBG strain is one order of magnitude lower as the expected chemical strain. This can be attributed to the viscoelastic behavior of the partially cured resin above its glass transition, allowing stress relaxation and limiting the interactions with the mold as well as with the optical fiber. The FBG signal is thus difficult to link to the actual chemical shrinkage. However, once the resin is cured, it is demonstrated that the FBG strain provides directly the CTE of the resin, taking into account its linear change with temperature. The effect of the degree of conversion 
on the CTE value was also emphasized from the different experiments.

\section{ACKNOWLEDGMENT}

The authors acknowledge C. Vergnes for his major contribution to the experimental work.

\section{REFERENCES}

1. N. Takeda and S. Ogihara, Compos. Sci. Technol., 52, 309 (1994).

2. F. Colpo, L. Humbert, and J. Botsis, Compos. Sci. Technol., 67, 1830 (2007)

3. M.J. O'Dwyer, G.M. Maistros, S.W. James, R.P. Tatam, and I.K. Partridge, Meas. Sci. Technol., 9, 1153 (1998).

4. M. Mulle, F. Collombet, P. Olivier, R. Zitoune, C. Huchette, F. Laurin, and Y.H. Grunevald, Compos. Part A, 40, 1534 (2009).

5. K. Magniez, A. Vijayan, and N. Finn, Polym. Eng. Sci., 52, 346 (2011).

6. A.L. Kalamkarov, S.B. Fitzgerald, and D.O. MacDonald, Compos. Part B, 30, 167 (1999)

7. Y. Okabe, S. Yashiro, R. Tsuji, T. Mizutani, and N Takeda, Compos. Part A, 33, 991 (2002).

8. M. Mulle, F. Collombet, P. Olivier, R. Zitoune, C. Huchette, F. Laurin, and Y.-H. Grunevald, Compos. Part A, 40, 94 (2009).

9. E. Marin, L. Robert, S. Triollet, and Y. Ouerdane, Polym. Test., 31, 1045 (2012).

10. D. Karalekas, J. Cugnoni, and J. Botsis, Compos. Part A, 39, 1118 (2008).

11. P. Bartolomeo, J.F. Chaillant, and J.K.L. Vernet, J. Polym. Sci. B, 38, 2154 (2000).
12. J.D. Russel, M.S. Genidy, M.S. Madhukar, and A.Y. Lee, $J$. Compos. Mater., 34, 22 (2000).

13. A. Othonos and K. Kalli, Fiber Bragg Gratings: Fundamentals and Applications in Telecommunications and Sensing, Artech House, hardcover, 443 (1999). ISBN 0-89006-344-3.

14. X. Tao, L. Tang, W. Du, and C. Choy, Compos. Sci. Technol., 60, 657 (2000).

15. J.A. Guemes and J.M. Menéndez, Compos. Sci. Technol., 62, 959 (2002).

16. Y.J. Rao, Opt. Laser Eng., 31, 297 (1999).

17. Y. Zhao and Y. Liao, Opt. Laser Eng., 41, 1 (2004).

18. S. Triollet, L. Robert, E. Marin, and Y. Ouerdane, Meas. Sci. Technol., 22, 015202 (2011).

19. M. Lai, D. Karalekas, and J. Botsis, Sensors, 13, 2631 (2013).

20. L. Robert and G. Dusserre, Polym. Eng. Sci. doi: 10.1002/ pen.23596 (2013). [Epub ahead of print].

21. D. Lecointe, "Caractérisation, simulation des processus de transferts lors d'injection de résine pour le procédé RTM," PhD Thesis, Univ. de Nantes, France, (1999).

22. P.I. Karkanas and I.K. Partridge, J. Appl. Polym. Sci., 77, 1419 (2000).

23. P.I. Karkanas and I.K. Partridge, J. Appl. Polym. Sci., 77, 2178 (2000).

24. X.A. Aduriz, C. Lupi, N. Boyard, J.L. Bailleul, D. Leduc, V. Sobotka, N. Lefèvre, X. Chapeleau, C. Boisrobert, and D. Delaunay, Compos. Sci. Technol., 67, 3196 (2007).

25. D. Lecointe, M. Villière, S. Nakouzi, V. Sobotka, N. Boyard, F. Schmidt, and D. Delaunay, Key Eng. Mater., 504, 1091 (2012).

26. T. Hobbiebrunken, B. Fiedler, M. Hojo, S. Ochiai, and K. Schulte, Compos. Sci. Technol., 65, 1626 (2005). 